\title{
Rapid recurrence of anaplastic carcinoma: advocacy towards non-surgery
}

\begin{abstract}
Anaplastic carcinoma of the thyroid (ATC) is a rare but aggressive tumor, its prognosis is dark. Most patients diagnosed with this disease are 65years of age or older, treatment is based on the combined action of radiotherapy, chemotherapy and surgical removal when possible. We report a case of anaplastic carcinoma of the thyroid which has been thundering in postoperative surgery after total thyroidectomy, this case highlights the ineffectiveness of surgery in the reduction of tumor mass. Surely contribute to a historical recurrence and aggravate the prognosis. We will study through a review of the literature the clinical aspects and discuss the therapeutic modalities of this cancer.
\end{abstract}

Keywords: anaplastic thyroid carcinoma, therapeutic modalities
Volume 5 Issue 5 - 2017

\author{
Habra B, El Mghari G, El Ansari N \\ Department of Endocrinology Diabetology and Metabolic \\ Diseases, CHU Mohamed VI Marrakech, Morocco
}

Correspondence: Bahia Habra, Department of Endocrinology Diabetology and Metabolic Diseases, CHU Mohamed VI Marrakech service d' endocrilnologie CHU Mohamed VI Marrakech Marocco, Tel 212672520912,

Emailb.habra2013@gmail.com

Received: July 14, 2017 | Published: October 13, 2017
Abbreviations: ATC, anaplastic thyroid cancer; AJCC, american joint commission on cancer; IMRT,intensity-modulated radiation therapy

\section{Introduction}

Anaplastic thyroid cancer (ATC) is one of the most aggressive solid tumors to affect humans, with a median survival on the order of 3 to 5 months following diagnosis. ${ }^{1}$ One-year and 10-year survival rates are estimated at $10-20 \%$ and less than 5\%, respectively, though some reports question the reliability of diagnoses in these longterm survivors. ${ }^{1,2}$ Recent advances in understanding the genetic and molecular pathogenesis of ATC hold promise for targeted therapy for this disease. ATC is usually resistant to standard chemotherapy. There are several clinical trials underway with small molecule tyrosine kinase inhibitors, antiangiogenesis agents and vascular-disrupting agents which might offer more drugs in the therapeutic armamentarium to combat this disease. ${ }^{1}$

\section{Note}

We report the case of the 44-year-old patient, who for 5years showed a cervical swelling gradually increasing in volume and subsequently compressive, with an alteration in the general condition, with cervical ultrasound completed by cervical CT In preoperative procedures: nodular heterogeneous hypoechoic goitre of $38 * 49 * 4 \mathrm{~mm}$ with calcifications $4 \mathrm{C}$ (according to the 2015 criteria of the American Cancer Society), with compression and repression of left jugular carotid and trachea, absence of cervical adenopathies (Figure 1). The gesture consisted of a total thyroidectomy, the anatomopathological study showed a malignant proliferation, little differentiated and infiltrating, presence of vascular emboli, peri-nervous entrapment and capsular effraction, the immunohistochemical study showed a carcinoma Little differentiated and infiltrating, classified pT3NxMx, the evolution was marked at day 5 of postoperative by recurrence of the cervical swelling with compressive signs to the type of dysphagia and dysphonia, the clinical examination finds: a firm anterior cervical mass painful An ultrasound was performed in which the bulk of the bilateral clavicular and bilateral cerebral regions that could be associated with lymphadenopathy were examined, a cervical computed tomography (Figure 2) had shown a large tumorous process from the cervico-mediastinal orifice to the hyoid bone infiltrating the soft tissue without adenopathy, after three days of development the patient died as a result of Complications and tumor hemorrhage.

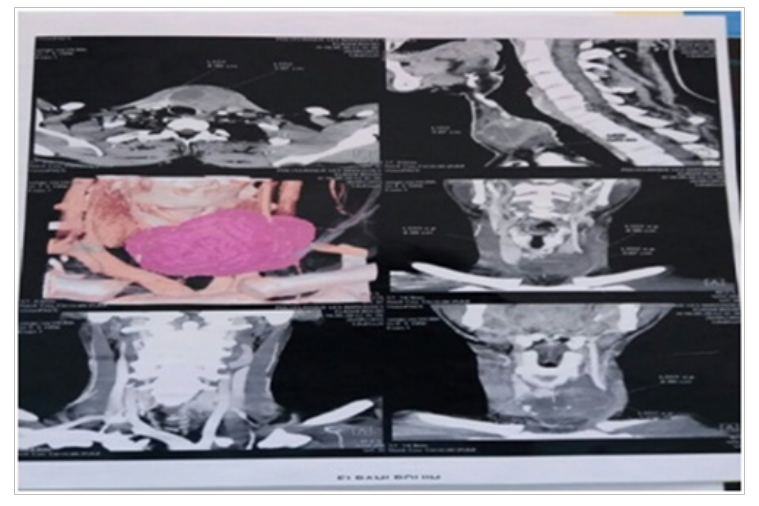

Figure I CT scan (before surgery) showing a voluminous cervical tumor process.

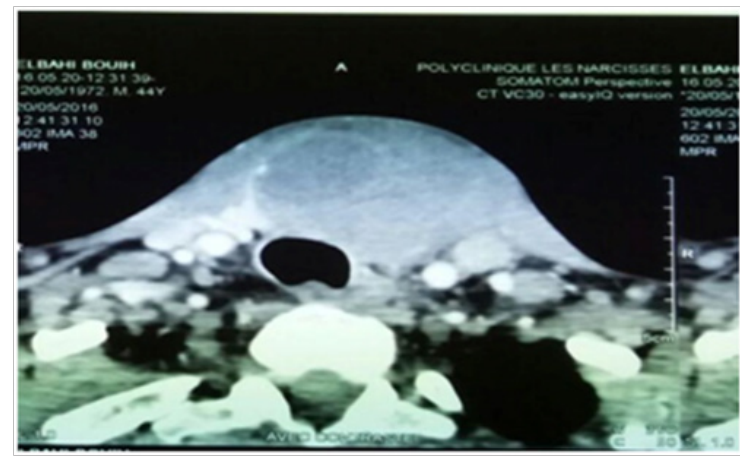

Figure $2 \mathrm{CT}$ scan showing a large cervical tumor process infiltrating soft tissues in relation to recurrence. 


\section{Discussion}

The majority of anaplastic cancers are revealed by the rapid transformation of an old goitre: increase in volume, pain, dysphonia, dyspnea by tracheal compression and dysphagia thereafter. The nodal invasions, the repercussion on the general state, immediately sign the gravity of the situation. The cervical ultrasound is not specific but allows suspecting a malignant tumor, reveals a lesion of thyroid origin, often totolobar, poorly limited and invading the extrathyroid tissues. The echostructure is mostly heterogeneous, predominantly hypoechoic, sometimes calcified in the case of pre-existing goitre. Vascularization varies according to the extent of tumor necrosis. ${ }^{3}$ In addition, it detects sub-clinical adenopathies and appreciates the local invasion of the tumor. ${ }^{4}$

In the literature, Anaplastic thyroid carcinoma is exceptional before the age of 40years, it occurs mainly in elderly subjects with a peak frequency of 60 to 70 years. Female predominance is reported in all series. ${ }^{5-7}$ Anaplastic carcinoma of the thyroid is usually found by voluminous goitre or a rapidly evolving old nodule. ${ }^{8-10}$

The compressive disorders associated with the tumor volume impose above all the practice of a rescue tracheotomy with thyroid biopsy confirming the anaplastic nature of the tumor. According to the study by Voutilainen. ${ }^{4} 33$ patients with anaplastic thyroid carcinoma, $30 \%$ required an emergency tracheotomy. The cytological and even histological diagnosis of anaplastic carcinoma of the thyroid is not always easy, thus posing several differential diagnoses such as thyroiditis, insular carcinoma, medullary carcinoma, thyroid lymphomas and thyroid metastases of another carcinoma, ${ }^{11-13}$ hence the importance of the immunohistochemical study in the diagnosis of this tumor which makes it possible to detect the presence of FAS proteins and cytokeratin and the negativity of the other classical tumor markers of thyroid cancers (thyroglobulin, Calcitonin, ACE). ${ }^{14}$ In our patient immunohistochemical study after fixation and inclusion in paraffin shows: intensive and focal cytoplasmic expression of the tumor cells of the anticytokine antibody (clone AE1/AE3, DAKO) with negativity of the other conventional tumor markers.

\section{Supported}

Management should be rapid for two reasons: the tumor doubling time is extremely short, and the extent of disease extension affects treatment options and survival. ${ }^{15}$ No standard support protocol has been established. The therapeutic approach consists of a multimodal strategy combining surgery, hyperfractioned and accelerated cervicomédiastinal external radiotherapy, and chemotherapy essentially based on doxorubicin, according to several possible sequences. $^{3}$

\section{General therapeutic approach}

Patients with ATC even in the absence of metastatic disease are considered to have systemic disease at the time of diagnosis. All ATCs are considered stage IV by the International Union against Cancer (UICC)-TNM staging and American Joint Commission on Cancer (AJCC) system. Multimodality treatment consisting of surgery when feasible combined with radiation and chemotherapy is generally recommended. The surgery must not compromise the functional anatomy of the cervical structures. The most common cause of death is invasion of vital local structures in the neck. Consequently, achieving good local control with surgery and or radiation confers short term palliative and survival benefit. Radiotherapy alone and in combination with surgery has been shown to achieve good local control in many studies. There is no consensus on the sequence of radiation and surgery.

\section{Radiotherapy}

The purpose of radiotherapy is diverse. It is proposed for curative purposes when it is used as adjuvant or neoadjuvant in addition to surgical treatment. It is also used as a first-line treatment for subsequent surgery on a surgically unaffordable tumor volume. ${ }^{16}$ Finally it can be performed in the emergency, palliative, with a simplified treatment plan in front of major compressive signs. ${ }^{3}$

Radiation does not alter the course of ATC in most patients. On the other hand, when combined with surgery and chemotherapy, it can prolong the short-term survival in select and subset of patients. Intensity-modulated radiation therapy (IMRT) based on computerized treatment planning and delivery is able to generate a dose distribution that delivers radiation accurately with sparing of the surrounding normal tissue. ${ }^{17,18}$ A report from MD Anderson Cancer Center compared outcomes for ATC patients treated with conformal 3-dimensional radiotherapy (3DRT) or IMRT. Of the 53 consecutive patients were analyzed, 31 patients were irradiated with curative intent with a median radiation dose of 55Gray (Gy; range, 4-70 Gy). Thirteen patients received IMRT to a median 60Gy (range, 39.9-69.0Gy). The majority of patients received chemotherapy with radiation The Kaplan-Meier estimate of overall survival (OS) at 1 year for definitively irradiated patients was $29 \%$. Five patients without distant metastases had no evidence of disease at last followup. Use of IMRT versus 3DRT did not influence toxicity. ${ }^{19}$

Toxicity can be a limiting factor with radiation, particularly, pharyngoesophagitis and tracheitis. Wong and coworkers also noted skin changes, esophageal toxicity, and radiation myelopathy. ${ }^{20}$ Daily doses of greater than $3 \mathrm{~Gy}$ should be cautiously used as it can increase the incidence of myelopathy. ${ }^{20}$ Wong and coworkers also noted skin changes, esophageal toxicity, and radiation myelopathy. ${ }^{20}$ Hyperfractionated radiation regimens delivering a total of around 46Gy have been most effective both with and without doxorubicin. ${ }^{1}$

\section{Chemotherapy}

It has been found that in vitro, anaplastic cell lines express less multiple drug resistance (mdr1) mRNA and P-glycoprotein while expressing more multidrug resistance-associated protein (MRP). ${ }^{21,22}$ This could explain the almost uniformly poor outcomes with chemotherapy. Doxorubicin is the most common agent used. A literature review by Ahuja showed a response rate around $22 \%$ for doxorubicin. ${ }^{23}$

Swaak-Kragten and others did a retrospective analysis of seventyfive ATC-patients treated between 1972 and 2003 in the Netherlands. Thirty-six patients underwent up-front surgery of which with $53 \%$ had a R0(negative microscopic resection margin)/R1(positive microscopic resection margin) resection.

According to Kim, a better local response is observed when using poly-chemotherapy than in mono-chemotherapy. This response is further enhanced when hyperfractionated radiotherapy is combined..$^{13}$ On the other hand, some authors mention that the indication of chemotherapy is limited by its toxicity, especially in elderly or multitared subjects. ${ }^{11,21,24}$ In a study from Serbia, between 1997 and 2007, 16 inoperable patients were treated with radiotherapy at $60 \mathrm{~Gy}$ followed by doxorubicin $60 \mathrm{mg} / \mathrm{m}^{2}$ and cisplatin $40 \mathrm{mg} / \mathrm{m}^{2}$ every 3 weeks. The 
overall response rate (ORR) was 25\% (95\% CI: 7-55). No toxic deaths occurred or grade 4 adverse events were reported after radiotherapy. Grade 4 toxicity was seen in 3 patients after chemotherapy. Mean patient OS was 12.33 months (95\% CI: 9.09-15.56) and median OS 11.0 months (95\% CI: 8.56-13.44). ${ }^{2}$ Shimaoka and colleagues reported three complete and three partial responders out of 19 patients treated with doxorubicin and cisplatin. ${ }^{25}$

Paclitaxel was found to have a response rate of 53\% $(95 \%$ confidence interval: $29-76 \%$ ) with one complete response and nine partial responses in 19 evaluable patients in a study conducted by Ain and colleagues. ${ }^{26}$ A nonconventional response rate definition was utilized in this study. A recent study done in Japan compared overall survival after induction chemotherapy by weekly paclitaxel administration for patients with stage IVB (nine patients) and IVC (four patients) disease with that of ATC patients with stage IVB $(\mathrm{n}: 5)$ and IVC (n:13) treated without paclitaxel. Complete response was seen in one, and two demonstrated partial response in the stage IVB group and one patient showed PR in stage IVC. After paclitaxel, curative surgery and adjuvant therapy were performed for four patients with stage IVB. All four patients were reported to be alive and disease-free 32 months after treatment. All four patients with stage IVC died of carcinoma within 8months. Overall survival of stage IVB patients with induction chemotherapy was better $(\mathrm{P}=0.0213)$ than that without the chemotherapy and also better $(\mathrm{P}=: 0.0467)$ than those with chemotherapy other than paclitaxel. However, induction chemotherapy did not improve the overall survival of stage IVC patients $(\mathrm{P}=0.2002){ }^{27}$ Docetaxel was administered intravenously at a dose of $60 \mathrm{mg} / \mathrm{m}^{2}$ over the course of $1 \mathrm{~h}$ every 3 weeks in seven patients with ATC who had received no prior chemotherapy in recent single center study in Japan. One patient had complete response, two patients had stable disease, and four patients had progressive disease. The response rate was $14 \%$, and the disease control rate (complete/ partial response plus stable disease) was $43 \%$. The median time to progression was 6 weeks (range, 1-50). The authors report that the toxicity was tolerable. ${ }^{28}$

\section{Surgery}

The place of surgical treatment is controversial: essential when the removal of the tumor can be complete in a patient able to receive upstream or downstream chemoradiotherapy treatment. Similarly, small anaplastic cancers discovered incidentally and whose treatment Surgery appears at first complete may present local relapses or a metastatic evolution..$^{10}$ Often radical surgery is impossible, limited to a simple biopsy or palliative surgery to reduce the speed and importance of locoregional extension as well as the metastatic power of this tumor. In the case of a locally invasive tumor deemed immediately inextricable, a first chemoradiotherapy could make it possible to select the patients in whom second surgical treatment can be beneficial. The theoretical advantage is to limit the development of metastases very early by systematic treatment, to increase the possibilities of a complete excision if an objective tumor response is obtained. ${ }^{29,30}$ For some authors, even in the metastatic disease stage, tumor reduction surgery associated with radiochemotherapy may be proposed in some patients for palliative purposes, in order to avoid death by asphyxia. ${ }^{16}$

Studies have shown that a trial of primary radiation therapy followed by surgical excision is currently feasible in order to better control the tumor locally. ${ }^{29,31,32}$

\section{Combined treatments}

In most institutional series, patients receiving (or in a position to receive) the combination of different treatments benefit from better local control over their disease and prolonged survival. ${ }^{3}$ In a study conducted at the MD Anderson Cancer Center, 12 of the 121 patients had survived more than two years: total thyroidectomy with complete macroscopic ablation of their tumor followed by postoperative chemoradiotherapy for $10 \mathrm{~d}$ Among them. ${ }^{3,33}$ This therapeutic curative strategy is especially beneficial for patients with a low tumor mass at the time of diagnosis: their survival was 43 months in the Haigh study, to be compared to the three months of median survival of patients who received a therapeutic approach Palliative approach. ${ }^{30}$ In some chemoresistant or radiolabeled tumors the disease progresses rapidly under treatment, some patients are not able to benefit from these therapeutic combinations because of a major deterioration of the general condition as soon as the diagnosis. Nevertheless, palliative management of patients with thyroid anaplastic cancer is often necessary.

\section{Emerging therapies}

As more data becomes available regarding the molecular pathogenesis of ATC, more targeted therapies are appearing in the clinic. Two of the most promising class of agents are the smallmolecule tyrosine-kinase angiogenesis inhibitors and vascular disrupting agents. There are two small-molecule tyrosine-kinase inhibitors in the midst of Phase II clinical trials including imatinib mesylate (Gleevac, Novartis, East Hanover, NJ, USA) and sorafenib (Nexavar, Onyx, San Fransisco, Calif, USA). Imatinib mesylate is an orally available selective c-abl tyrosine-kinase inhibitor. It has been shown effective in vitro in anaplastic thyroid cell lines. ${ }^{34,35}$ Another study did not find it effective. ${ }^{36}$ Sorafenib is a novel small-molecule tyrosine-kinase inhibitor which acts on the raf-1 serene/threonine kinase. BRAF mutations are thought to be an important event in the evolution of ATC and are a potential therapeutic target for treatment. Sorafenib also blocks the receptor tyrosine kinases to the vascular endothelial growth factor receptor 2 (VEGFR2) and platelet-derived growth factor receptor $\beta$ (PDGFR- $\beta$ ) and thus has antiangiogenesis properties as well. Sorafenib inhibited the growth of rat orthotopic ATC xenografts, and the survival of test animals was improved in recently reported preclinical study. ${ }^{37}$ Axitinib (AG-013736) is an oral, selective inhibitor of VEGFRs 1, 2, and 3, and preclinical studies show that it blocks angiogenesis and tumor blood flow in preclinical models. ${ }^{38}$ Cohen and colleagues studied this agent in a clinical trial on patients with advanced thyroid cancer. Two of the 60 patients had ATC. One patient has a partial response, and the other one progressed in spite of treatment. ${ }^{39}$

Fosbretabulin is a derived from the African bush willow, Combretum caffrum. It is a novel tubulin-binding, vascular=disrupting agent and should be differentiated from the angiogenesis inhibitors discussed above. It displays potent and selective toxicity towards tumor vasculature and is thought to act by endothelial disruption of established tumor vasculature. ${ }^{40,41}$ The agent binds avidly to tubulin at the colchicine-binding site to inhibit microtubule assembly and destabilizes the cytoskeleton. ${ }^{42}$ Fosbretabulin has also been shown to enhance or act synergistically with radiation and several chemotherapeutic agents..$^{43,44}$

In a Phase II study in 26 patients with ATC, this agent was found to be well tolerated with grade 3 or greater toxicity being observed 
in $35 \%$ of patients. Median survival was 4.7 months, with $34 \%$ and $23 \%$ alive at 6 and 12 months, respectively. Median duration of stable disease was 12.3months (range 4.4-37.9). Low-baseline soluble intracellular adhesion molecule-1 (sICAM) appeared to predict better event-free survival. ${ }^{45}$ In a Phase $2 / 3$ trial, fosbretabulin, paclitaxel, and carboplatin combination was compared to carboplatin and paclitaxel only in 80 patients with ATC. Interim results have been presented at the European Society of Medical Oncology meeting in Milan, Italy (October 2010). Preliminary results showed that the combination is well tolerated and showed an improvement in overall survival from 4.1 months to 5.1 months, with hazard ration of 0.71 . Value could not be calculated, as the study had to be truncated due to poor accrual. Oneyear survival was almost doubled with fosbretabulin, when compared to chemotherapy alone ( $23 \%$ versus $9 \%)$. OS was objectively longer in patients less than 60 years of age, increasing from a median of 3.1 months to 10.9 months (HR of $0.38,95 \% \mathrm{CI}$ : $0.16,0.88) .{ }^{46-50}$

\section{Prognosis}

The best prognostic factors are: age less than 60 years, female sex, tumor less than $5-6 \mathrm{~cm}$, localized disease, and general condition. ${ }^{3}$ In all retrospective studies, metastatic extension with an acute cervical array as well as the existence of inflammatory signs appears to be the most unfavorable prognostic elements. The average survival is four to nine months. In our case witness, the young age was not in favor, time more than the modality of care. The dramatic mortality of anaplastic cancer of the thyroid gland especially in the case of metastasis and the inadequacy of conventional therapies impose the search for new therapeutic pathways. Therapeutic applications of targeted approaches are entering thyroid oncology with promising results. Some treatments are aimed at restoring cell differentiation, blocking the action of abnormally activated oncogenes. Others specifically inhibit the abnormally overexpressed EGF receptor or tumor angiogenesis. The published works concern therapeutic agents still in development or already commercialized. ${ }^{3}$ Many are expected to be derived from combrestatin A4 derivatives, tyrosine kinase inhibitors and farnesyl transferase inhibitors, which have shown promising results at the experimental stage and whose place and mode of use will remain to be defined in comparison with conventional therapies.

\section{Conclusion}

The prognosis of these cancers, especially in the case of metastases, nevertheless remains bleak. Only patients in the stage of localized disease can hope for better survival at the cost of heavy treatment. This is why the importance of rapid therapeutic implementation in specialized settings must be emphasized. The inadequacy of conventional therapies impose the search for new therapeutic pathways. There has not been much improvement in response rates achieved above the $20 \%$ response rate seen with doxorubicin over the years. Further research is needed to evaluate new treatments for this almost uniformly fatal disease. ${ }^{1}$

\section{Acknowledgments}

None.

\section{Conflicts of interest}

The authors declare that there are no conflicts of interest.

\section{Funding}

None.

\section{References}

1. Nagaiah G, Hossain A, Mooney CJ, et al. Anaplastic thyroid cancer: a review of epidemiology, pathogenesis, and treatment. $J$ Oncol. 2011;2011:542358.

2. Are C, Shaha AR. Anaplastic thyroid carcinoma: biology, pathogenesis, prognostic factors, and treatment approaches," Annals of Surgical Oncology. 2006;13(4):453-464.

3. Wemeau JL, Do Cao C. Anaplastic cancers of the thyroid gland Department of endocrinology, metabolic diseases, endocrinological clinic Marc-Linquette. Lille CHRU, 6, rue du Professeur-Laguesse, 59037 Lille cedex, France. 2000.

4. Lakhloufi A, Moulay Rachid M, Khaiz D, et al. Anaplastic cancer of the thyroid. Medicine of the Maghreb. 1998;70:11-17.

5. Voutilainen PE, Multanen M, Haapianinen RK, et al. Anaplastic thyroid carcinoma survival. World J Surg. 1999;23:975-979.

6. Rodriguez JM, Pinero A, Ortiz S. Clinical and histological differences in anaplastic thyroid carcinoma. Eur J Surgery. 2000;166(1):34-33.

7. Besic N, Auersperg M, Us-Krasovec. Effect of primary treatment on survival anaplastic thyroid carcinoma. Eur $J$ Surg Oncol. 2001;27(3):260-264.

8. Schlumberger MJ. Anaplastic thyroid carcinoma. Orphanet encyclopedia. $2005 ; 1-4$.

9. Goutsouliac V, Hay JH. Anaplastic thyroid cancer in British Columbia 1985- 1999: A population-based study. Clin Oncol. 2005;17(2):75-78.

10. Bittmann O, Bruneton JN, Fenart D. Imaging of anaplastic carcinoma of the thyroid gland. J Radiol. 1992;73:35-38.

11. Sugito K, Ito K, Mimura T. The important role of thyroid carcinoma in the management of anaplastic. Surgery. 2002;131(3):245-248.

12. Lam K, Lo C, Chan K. Insular and anaplastic carcinoma of the thyroid: a 45-year comparative study at a single institution and a review of the thyroid significance of p53 and p21. Ann Surg. 2000;231(3):329-338.

13. Kim JH, Leeper RD. Doxorubicin and radiation therapy. Cancer. 1987;60:2372-2375.

14. Sckiguchi M, Shiroko Y, Arai T. Biological charateristics and chemosensitivity Profile of human anaplastic thyroid carcinoma cell lines. Biomed Pharmacother. 2001;55(8):466-474.

15. Pasieka JL.Anaplastic thyroid cancer. Curr Opin Oncol.2003;15(1):78-83.

16. De Besi P, Busnardo B, Toso S, et al. Combined chemotherapy With bleomycin, adriamycin, and platinum in advanced thyroid cancer. $J$ Endocrinol Invest. 1991;14(6):475-80.

17. Lee N, Puri DR, Blanco AI, et al. Intensity-modulated radiation therapy in head and neck cancers: an update. Head \& Neck. 2007;29(4):387-400.

18. Rosenbluth BD, Serrano V, Happersett L. Intensity-modulated radiation therapy for the treatment of nonanaplastic thyroid cancer. International Journal of Radiation Oncology Biology Physics. 2005;63(5):1419-1426.

19. Bhatia A, Rao A, Ang KK, et al. Anaplastic thyroid cancer: clinical outcomes with conformal radiotherapy. Head Neck. 2010;32(7):829-836.

20. Wong CS, Van Dyk J, Simpson WJ. Myelopathy following hyperfractionated accelerated radiotherapy for anaplastic thyroid carcinoma. Radiotherapy and Oncology. 1991;20(1):3-9.

21. Lehnert M. Clinical multidrug resistance in cancer: a multifactorial problem. Eur J Cancer. 1996;32A(6):912-920.

22. Shoji S, Sugawarab I, Masatoshi W, et al. Lack of a point mutation of human DNA topoisomerase II in multidrug-resistant anaplastic thyroid carcinoma cell lines. Cancer Letters. 1997;116(1):33-39. 
23. Ahuja S, Ernst H. Chemotherapy of thyroid carcinoma. Journal of Endocrinological Investigation. 1987;10(3):303-310.

24. Ohtsuru A, Podtcheko AP, Tsuda S. A novel radiosensitizing therapy for anaplastic thyroid cancers. International Congress Series. $2003 ; 1258: 273-276$

25. Shimaoka K, Schoenfeld DA, DeWys WD, et al. A randomized trial of doxorubicin versus doxorubicin plus cisplatin in patients with advanced thyroid carcinoma. Cancer. 1985;56(9):2155-2160.

26. Ain KB, Egorin MJ, DeSimone PA. Treatment of anaplastic thyroid carcinoma with paclitaxel: phase 2 trial using ninety-six-hour infusion. Collaborative Anaplastic Thyroid Cancer Health Intervention Trials (CATCHIT) Group. Thyroid. 2000;10(7):587-594.

27. Higashiyama T, Ito Y, Hirokawa M, et al. Induction chemotherapy with weekly paclitaxel administration for anaplastic thyroid carcinoma. Thyroid. 2010;20(1):7-14.

28. Kawada K, Kitagawa K, Kamei S, et al. The feasibility study of docetaxel in patients with anaplastic thyroid cancer. Jpn J Clin Oncol. 2010;40(6):596-599.

29. Lo C, Lam K, Wan KY. Anaplastic carcinoma of the thyroid. Surgery. 1999;177(4):337-339.

30. Venkatesh YS, Ordonez NG, Schultz PN, et al. Anaplastic carcinoma of the thyroid. A clinicopathologic study of 121 cells. Cancer. 1990;66(2):321-330.

31. Marc D, Posner M, Jeanne M. Dose optimization for the treatment of anaplastic thyroid carcinoma: a comparison of treatment planning techniques. Int J Radiat Oncol. 2000;48(2):475-83.

32. Tennvall J, Lundell, Wahlberg P. Anaplastic thyroid carcinoma: Three protocols combining Doxorubicin, hyperfractionated radiotherapy and surgery. Br J Cancer. 2002;86(12):1848-1853.

33. De Crevoisier R, Baudin E, Bachelot A, et al. Combined treatment of anaplastic thyroid carcinoma with surgery, chemotherapy, and hyperfractionated accelerated external radiotherapy. Int J Radiat Oncol Biol Phys. 2014;60(4):1137-1143.

34. Podtcheko A, Ohtsuru A, Namba H. Inhibition of ABL tyrosine kinase potentiates radiation-induced terminal growth arrest in anaplastic thyroid cancer cells. Radiation Research. 2006;165(1):35-42.

35. Podtcheko A, Ohtsuru A, Tsuda S, et al. The selective tyrosine kinase inhibitor, STI571, inhibits growth of anaplastic thyroid cancer cells. $J$ Clin Endocrinol Metab. 2003;88(4):1889-1896.

36. Dziba JM, Ain KB. Imatinib mesylate (gleevec; STI571) monotherapy is ineffective in suppressing human anaplastic thyroid carcinoma cell growth in vitro. Journal of Clinical Endocrinology \& Metabolism. 2004;89(5):2127-2135.

37. Kim S, Yazici YD, Calzada G, eta al. Sorafenib inhibits the angiogenesis and growth of orthotopic anaplastic thyroid carcinoma xenografts in nude mice. Mol Cancer Ther. 2007;6(6):1785-1792.
38. Inai $\mathrm{T}$, Mancuso M, Hashizume $\mathrm{H}$, et al. Inhibition of vascular endothelial growth factor (VEGF) signaling in cancer causes loss of endothelial fenestrations, regression of tumor vessels, and appearance of basement membrane ghosts. Am J Pathol. 2004;165(1):35-52.

39. Cohen EE, Rosen LS, Vokes EE, et al. Axitinib is an active treatment for all histologic subtypes of advanced thyroid cancer: results from a phase II study. J Clin Oncol. 2008;26(29):4708-4713.

40. Dark GG, Hill SA, Prise VE, et al. Combretastatin A-4, an agent that displays potent and selective toxicity toward tumor vasculature. Cancer Res. 1997;57(10):1829-1834.

41. Vincent L, Kermani P, Young LM, et al. Combretastatin A4 phosphate induces rapid regression of tumor neovessels and growth through interference with vascular endothelial-cadherin signaling. Journal of Clinical Investigation. 2005;115(11):2992-3006.

42. Pettit GR, Singh SB, Hamel E, et al. Isolation and structure of the strong cell growth and tubulin inhibitor combretastatin A-4. Experientia. 1989;45(2):209-211.

43. Grosios K, Loadman PM, Swaine DJ, et al. Combination chemotherapy with combretastatin A-4 phosphate and 5-fluorouracil in an experimental murine colon adenocarcinoma. Anticancer Research. 2000;20(1):229-233.

44. Murata R, Siemann DW, Overgaard J, et al. Interaction between combretastatin A-4 disodium phosphate and radiation in murine tumors. Radiother Oncol. 2001;60(2):155-161.

45. Mooney CJ, Nagaiah G, Fu P, et al. A phase II trial of fosbretabulin in advanced anaplastic thyroid carcinoma and correlation of baseline serum-soluble intracellular adhesion molecule-1 with outcome. Thyroid. 2009;19(3):233-240.

46. Sosa JA, Elisei R, Jarzab B. Randomized phase $2 / 3$ trial of a tunor vascular disrupting agent, fosbertubulin (CA4P), with carboplatin (C), and paclitaxel $(\mathrm{P})$ in anaplastic thyroid cancer (ATC): interim safety and efficacy results of the FACT trial. Journal of Clinical Oncology. 2010;29(15):5502-5503.

47. Pierie JP. The effect of surgery and radiotherapy on the outcome of anaplastic thyroid carcinoma. Ann surg oncol. 2002;9(1):57-64.

48. McIver B, Hay ID, Giuffrida DF. Anaplasic thyroid carcinoma: A50-year experience at single institution. Surgery. 2001;130(6):1028-1034.

49. Haigh PI, Ituarte PH, Wu HS, et al. Completely resected Anaplastic thyroid carcinoma combined with adjuvant chemotherapy and irradiation isassociated with Prolonged survival. Cancer. 2001;91(12):2335-2342.

50. ASwaak Kragten AT, de Wilt JH, Schmitz PI, et al. Multimodality treatment for anaplastic thyroid carcinoma-treatment outcome in 75 patients. Radiotherapy and Oncology. 2009;92(1):100-104. 